\title{
Rapport au travail des enseignants de judo en France : entre valeurs défendues et tensions inhérentes à l'activité
}

French judo instructors' perception of work: the relationship between the values promoted and the tensions inherent to the activity

Thérèse Perez-Roux, Sylvie Perez, Philippe Gabriel, Jacqueline Papet et Florence Cassignol

\section{OpenEdition}

Journals

Édition électronique

URL : http://journals.openedition.org/activites/3741

DOI : 10.4000/activites.3741

ISSN : $1765-2723$

Éditeur

ARPACT - Association Recherches et Pratiques sur les ACTivités

Référence électronique

Thérèse Perez-Roux, Sylvie Perez, Philippe Gabriel, Jacqueline Papet et Florence Cassignol, « Rapport au travail des enseignants de judo en France : entre valeurs défendues et tensions inhérentes

à l'activité », Activités [En ligne], 16-1 | 2019, mis en ligne le 15 avril 2019, consulté le 09 août 2019.

URL : http://journals.openedition.org/activites/3741 ; DOI : 10.4000/activites.3741

Ce document a été généré automatiquement le 9 août 2019

\section{(i) $(9)$}

Activités est mis à disposition selon les termes de la licence Creative Commons Attribution - Pas d'Utilisation Commerciale - Pas de Modification 4.0 International. 


\section{Rapport au travail des enseignants de judo en France : entre valeurs défendues et tensions inhérentes à l'activité}

French judo instructors' perception of work: the relationship between the values promoted and the tensions inherent to the activity

Thérèse Perez-Roux, Sylvie Perez, Philippe Gabriel, Jacqueline Papet et Florence Cassignol

\section{NOTE DE L'ÉDITEUR}

Article soumis le 3 juin 2018, accepté le 8 janvier 2019

\section{NOTE DE L'AUTEUR}

Enseignant-e-s : pour ne pas alourdir le texte, nous nous conformons à la règle qui permet d'utiliser le masculin avec la valeur de neutre.

\section{Contexte et problématique}

1 Les évolutions socio-économiques, idéologiques, politiques et les nouveaux enjeux qui leur sont liés affectent aujourd'hui les champs de l'enseignement, de la formation et de l'éducation, entendus dans leur pluralité. De fait, les politiques ministérielles (nationales et internationales) dans des domaines aussi divers que l'enseignement, la santé, le travail social, le sport, les loisirs et la culture entraînent inévitablement des effets sur le rapport au travail des acteurs de ces systèmes (Mazade \& Hinault, 2014 ; Perez-Roux \& Balleux, 
2014). Ces évolutions sont susceptibles de perturber, au moins pour un temps, les logiques antérieures et la manière d'appréhender la sphère professionnelle et ses contours. Elles sont aussi l'occasion de trouver des formes négociées (pour soi et pour autrui) permettant à la fois de prendre en compte les directives ministérielles/institutionnelles et de conserver ce qui semble essentiel dans son activité, en se saisissant des opportunités contextuelles, personnelles, sociales, dans une visée de développement professionnel.

2 L'étude s'intéresse au domaine du sport et plus particulièrement au monde de l'enseignement $\mathrm{du}$ judo, pris dans ces processus de changement et qui s'organise aujourd'hui entre deux dimensions: la première centrée sur le versant éducatif de l'activité ; la seconde organisée à partir du versant compétitif. Comme le développent des chercheurs dans le champ scolaire, on retrouve une double contrainte d'efficacité (ici sportive) et de démocratisation qui suppose une perspective éducative-intégrative (Dutercq, 2008; Dutercq \& Perez-Roux, 2011; van Zanten, 2010). Cette tension se double parfois d'un malentendu entre les choix politiques de la FFJDA ${ }^{1}$ et la construction d'une base de pratiquants actuels/futurs qui répond à des enjeux et besoins parfois éloignés des préoccupations de la sphère décisionnelle. Cette problématique oblige certains enseignants ${ }^{2}$ à reconfigurer leur activité pour répondre à des demandes/logiques parfois concurrentes, que celles-ci soient parentales, fédérales ou locales (subventions des municipalités, soutien logistique, accès aux infrastructures, reconnaissance de l'implication pour la collectivité, etc.).

3 Cette recherche mobilise différentes sources: a) elle s'appuie sur des travaux qui s'intéressent à l'activité professionnelle des acteurs du monde sportif (entraîneurs nationaux, conseillers techniques sportifs, éducateurs territoriaux (Collinet, Bernardeau, \& Moreau, 2009; Fleurance \& Perez, 2006, 2008, 2009; Perez, 2009) ; b) elle intègre des études visant à comprendre qui sont les enseignants de judo (étude SOFRES, 2001) et de quelle manière ces derniers défendent certaines valeurs du judo (Bergeret, 1983 ; Julhe, 2012) dans un contexte sociétal en évolution; c) elle prend en compte d'autres travaux qui mettent en lumière les grands organisateurs historiques, institutionnels, symboliques (Brousse, 2005), techniques et didactiques du judo (Bouthier, 2000 ; Loizon \& Martin, 2006 ; Terrisse, 1996) et permettent une ouverture sur les enjeux de son enseignement.

4 Les valeurs d'éducation et de formation du citoyen, largement défendues par les enseignants de judo, les contenus moteurs, mais aussi socio-affectifs et culturels privilégiés dans cette activité sportive, nous amènent à mobiliser des travaux conduits préalablement auprès des enseignants d'EPS (Perez-Roux, 2011), des enseignants du secondaire (Barrère, 2002; Tardif \& Lessard, 1999) et des entraîneurs nationaux (Fleurance \& Perez, 2006).

5 Dans cette contribution, nous nous intéressons plus particulièrement aux représentations et valeurs des enseignants de judo. Il s'agit de repérer comment, face à des évolutions qui influencent inévitablement leur pratique d'enseignement, ces derniers (re)construisent le sens de leur travail auprès de différents publics (enfants, adolescents, adultes). Entre normes institutionnelles, ressources pragmatiques ou symboliques mobilisées antérieurement et espaces de reconnaissance sociale, quelles dynamiques professionnelles peut-on repérer? Ces dynamiques sont fortement ancrées dans l'expérience subjective des individus, qu'elle soit marquée par le sentiment d'une injonction à s'adapter ou, à l'inverse, par celui d'une opportunité à saisir. La réflexion sur les problématiques que peuvent vivre ces enseignants (Balleux \& Perez-Roux, 2011; Perez-Roux \& Salane, 2013) nous conduit à: 1) préciser les caractéristiques socio- 
professionnelles de ce groupe ; 2) en comprendre les valeurs dominantes et plus ou moins partagées; 3) repérer un certain nombre de tensions qui traversent leur activité d'enseignant aujourd'hui, qu'elle soit bénévole ou rétribuée.

\section{Repères théoriques}

6 Au préalable, il semble important de préciser d'où nous partons lorsque nous employons le terme "activité ", mobilisé ici dans deux acceptions. Tout d'abord pour parler du travail et englober un ensemble d'éléments le structurant: représentations, valeurs, pratiques, modes d'engagement, etc. Ensuite, dans le contexte de notre étude, pour parler de l'activité sportive judo en tant que pratique sociale de référence. L'activité n'est donc pas investie à travers l'analyse des réalisations des individus en situation réelle, mais dans le sens développé par la sociologie des professions qui vise un triple objet: l'organisation sociale des activités de travail, la signification subjective de celles-ci et les modes de structuration des marchés du travail (Dubar \& Tripier, 1998).

7 Le cadre théorique de cette étude croise une orientation psycho-sociale (représentations, valeurs, implication professionnelle) et une orientation sociologique (rapport au travail, reconnaissance, identités professionnelles). Si les représentations sociales, en tant que formes de connaissances porteuses de valeurs, donnent sens à la pratique et légitiment une certaine vision du monde (Moscovici, 1961), les représentations professionnelles portent, quant à elles, sur des objets spécifiques, directement reliés à la sphère du travail, à ses enjeux, à ses pratiques, à ses relations, à ses imaginaires. Elles servent à agir et réagir face à l'environnement tout en permettant à l'individu de conserver un équilibre cognitif dans un contexte professionnel particulier. En ce sens, les représentations sont des matrices d'action qui contribuent à la construction des identités professionnelles.

8 Comprendre les représentations et les valeurs, c'est accéder, au moins en partie, à ce qui fonde et organise les pratiques. Cette approche peut venir compléter ou éclairer différemment celle(s) choisie(s) plus habituellement dans le champ de l'analyse de l'activité.

9 À ce titre, notre approche interroge les valeurs, à la fois parce qu'elles semblent organiser les choix personnels et professionnels des enseignants de judo et parce qu'elles se révèlent mobilisatrices pour l'action. En effet, la notion de valeur renvoie à une sorte d'absolu idéal, de critère ultime d'appréciation et de justification :

«la valeur n'est pas seulement principe de jugement, elle est aussi source d'émotion et principe d'action : la valeur nous mobilise, nous appelle, nous engage ; c'est une idée, mais en même temps une force, tout à la fois impérieuse et matérielle » (Forquin, 1994, p. 1026).

Les valeurs engagent donc l'individu de façon intense dans la vie personnelle, sociale et professionnelle. Pour les enseignants de judo, elles constituent sans doute l'un des fondements du rapport au travail. Car, comme le rappelle Forquin (1994) :

«s'il est vrai qu'enseigner suppose des efforts, des coûts, des sacrifices de toute nature, au sens propre du mot, il faut que ce qu'on enseigne vaille la peine. À côté des justifications d'opportunité et des justifications instrumentales (celles qui dépendent des contraintes et des circonstances), il doit exister aussi des justifications fondamentales, qui touchent à la valeur propre ou intrinsèque, de la chose enseignée » (p. 1027).

10 De ce point de vue, l'implication professionnelle peut être considérée comme un révélateur des conduites d'un sujet dans les rapports qu'il établit avec son environnement 
professionnel. Si les représentations professionnelles sont en lien avec la pratique, l'identité et les savoirs professionnels, l'implication surplombe ce système et en représente la ressource énergétique (Mias, 1998). Les acteurs privilégient des implications à l'intérieur d'un espace de travail, lorsqu'elles prennent sens pour eux et donnent une cohérence à leur action. Le degré de reconnaissance et le sentiment de contrôle de la situation qui en résultent peuvent conduire à des réorganisations partielles des représentations, des pratiques professionnelles et du système de valeurs des individus.

Dans une orientation sociologique, nous abordons le travail enseignant comme une expérience qui se vit au cœur des interactions humaines et génère un certain nombre de dilemmes professionnels (Tardif \& Lessard, 1999). Pour ces auteurs, dont les travaux portent sur les enseignants du système scolaire, il existe différentes façons de décrire et/ ou de comprendre le travail enseignant, dans sa complexité, en tenant compte à la fois de son versant codifié (prescriptions, contraintes formelles, normes, règlements, procédures, etc.) et de son versant flou (aspects implicites et invisibles du métier, contingences, imprévus). Le cadre développé nous semble pertinent pour aborder, dans une certaine mesure, le travail d'autres enseignants (statutairement éducateurs sportifs) qui interviennent hors du cadre scolaire. En effet, Tardif et Lessard envisagent ce travail comme " une construction sociale comportant de multiples facettes » (ibid, p. 29). Organisé sur la base de relations humaines diversifiées, il dépasse largement la seule transmission de savoirs, nécessite des tâches plurielles, suppose des ajustements importants (évolution des publics, attentes parentales, nouvelles prescriptions institutionnelles, etc.). Le côté composite du travail enseignant peut ainsi conduire à gérer des aspects hétérogènes, parfois contradictoires, renvoyant à des tensions ou à des dilemmes internes, qui engagent fortement la subjectivité des acteurs :

«A la fois statut et activité, le travail enseignant peut également être abordé, décrit et analysé en fonction de l'expérience du travailleur, c'est-à-dire du travail ${ }^{3}$ tel qu'il est vécu et signifié par et pour lui-même » (ibid, p. 40).

12 Cette étude s'intéresse donc au rapport au travail des enseignants de judo, entendu comme le « rapport affectif que les individus entretiennent avec les fonctions qu'ils exercent dans la sphère du travail » (Paugam, 2000, p. 43). En ce sens, la satisfaction que chacun y trouve permet de comprendre son degré d'engagement, souvent en lien avec des éléments gratifiants et stimulants. Dubar (2000) envisage ce rapport à travers le monde vécu du travail et l'espace d'identification qu'il permet:

«j'appelle sens du travail la composante des identités professionnelles qui concerne le rapport à la situation de travail, à la fois l'activité et les relations de travail,

l'engagement de soi dans l'activité et la reconnaissance de soi par les partenaires » (p. 104).

13 Nous faisons l'hypothèse que les situations de travail des enseignants de judo, extrêmement contrastées, nécessitent de nombreux ajustements aux contextes, aux publics, aux collectifs, ce qui inévitablement vient impacter leur pratique d'enseignement et la manière dont ils la définissent et la vivent. En effet, pour Charlot (1997), la notion de «rapport à » inclut "les représentations, envisagées comme des systèmes de relation, d'interprétation, ancrées dans un réseau de significations» (p. 93). C'est ce réseau de significations, en lien avec l'activité déclarée, que nous tentons de mettre au jour dans cette étude. 


\section{Méthodologie}

14 Le recueil de données s'est organisé en plusieurs étapes. Dans un premier temps, nous avons privilégié une entrée ethnographique (Cleret, 2013; Laplantine, 1996). Une immersion dans le monde des enseignants de judo hauts-gradés ${ }^{4}$ (La Londe les Maures, juin 2014) a permis de confirmer les enjeux de cette étude. Les traces de cette immersion ont été consignées dans un journal de recherche qui s'est appuyé sur des échanges lors de moments informels (notamment les repas), sur des observations pendant les séquences de formation ou d'information, sur des entretiens non directifs avec trois cadres de la fédération, complétés par un entretien avec un enseignant haut-gradé, dirigeant d'un club de plus de 300 licenciés. Cette première étape (juin 2014 - juin 2015) a été l'occasion de recueillir une première image de l'enseignement du judo en France aujourd'hui. Les échanges se sont progressivement orientés vers les problématiques rencontrées, les éventuelles évolutions de l'activité d'enseignant et d'entraîneur, les analyses de la situation contrastée des clubs, les positionnements des enseignants pris dans différentes logiques éducatives, compétitives, mais aussi socio-économiques.

L'enquête a été reprise et complétée entre mai et juin 2016 grâce à un élargissement de l'équipe de chercheurs. Des entretiens semi-directifs $(n=12)$ avec des enseignants de la région Languedoc-Roussillon et PACA ont permis: a) une forme d'acculturation des chercheurs ; b) la stabilisation d'un guide d'entretien ; c) l'élaboration d'un questionnaire à visée nationale.

16 En septembre 2016, 4 chercheurs ont participé à un stage de rentrée (grande région Occitanie) en immersion complète pendant deux jours. Lors de ce séjour, des entretiens semi-directifs (Blanchet \& Gotman, 2007 ; Kaufmann, 1996) ont été réalisés in situ $(\mathrm{n}=44)$ avec les enseignants présents, sur la base de critères diversifiés (âge, sexe, secteur rural/ urbain, temps plein/partiel, bénévole/salarié, enseignement dans un ou plusieurs clubs). Les entretiens ont été intégralement retranscrits et rendus anonymes.

Un questionnaire a par ailleurs été proposé aux participants de ce stage $(n=132)$. Il a été complété par une enquête nationale (octobre-novembre 2016) via le site Facebook des enseignants de judo $(n=108)$, ce qui donne un total de retours exploitables de 240 questionnaires. Composé de plusieurs parties, ce questionnaire a permis, dans un premier temps, à l'aide de questions fermées, de recueillir les caractéristiques personnelles et sociales (sexe, âge, situation familiale, nombre d'enfants, niveau d'études...), professionnelles (diplôme sportif, statut actuel, niveau de pratique du judo en compétition, ancienneté de pratique, salaire, participation à des stages et besoins en formation), et organisationnelles (publics, nombre de licenciés du club, environnement...) des enseignants de judo interrogés. Il a ensuite permis d'explorer différents thèmes comme :

- La satisfaction liée à l'activité d'enseignant de judo (échelle de Likert en 4 points de « Pas du tout » à « Tout à fait ») ;

- Les représentations professionnelles en termes de métiers jugés les plus en proximité avec l'enseignement du judo (question à choix multiples simple ; exemples d'items : éducateur, entraîneur, technicien, animateur...) et en termes de compétences jugées essentielles (échelle de Likert en 4 points; exemple d'items: un enseignant de judo est d'abord une personne qui... « enseigne des techniques », "prépare à la compétition », «transmet des valeurs ", etc.); 
- La place des outils numériques dans la pratique d'enseignement (échelle de Likert en 4 points et question ouverte);

- Les échanges entre vie de travail et vie hors travail (question fermée : ressources/obstacles/ sans rapport).

En croisant ces différentes données, nous nous proposons de revenir sur les valeurs partagées et sur les tensions auxquelles un certain nombre d'enseignants de judo sont confrontés dans leur activité au quotidien.

\section{Résultats}

\subsection{Les valeurs comme passeport, l'éducatif comme mission}

L'entrée dans le monde du judo nous amène à caractériser le groupe d'enseignants qui a répondu à l'enquête par questionnaire. Une étude réalisée en 2001 par la SOFRESSPONSORING pour la FFJDA, portant sur 409 professeurs de judo, rendait compte des caractéristiques de ce groupe professionnel. Certaines caractéristiques générales de l'activité des enseignants de judo ont pu être réaffirmées. Ainsi, le fait qu'enseigner le judo s'inscrit dans la durée, soit une activité très diversifiée avec des valeurs éducatives fortement revendiquées est une constante dans l'enseignement du judo. À l'inverse, les conditions d'enseignement très contrastées entre les enseignants, la nécessité d'une plus grande diversification des pratiques et un contexte sociétal plus difficile sont des caractéristiques qui ont fortement évolué au cours de ces 25 dernières années ${ }^{5}$.

\subsubsection{Une population non homogène et des conditions d'enseignement contrastées}

Les caractéristiques socio-biographiques du groupe des enseignants de judo ayant répondu à l'enquête se révèlent relativement contrastées. Les enquêtés sont majoritairement des hommes (83\%). L'activité d'enseignement se déploie sur des temporalités relativement longues: si $30 \%$ des enseignants de judo sont âgés de 20 à 35 ans, $53 \%$ ont entre 36 à 55 ans, $18 \%$ entre 56 ans et plus. Sur le plan du statut, $53 \%$ des enseignants sont bénévoles, $28 \%$ salariés et $19 \%$ indépendants.

21 Les résultats mettent en relief des conditions de travail hétérogènes: si $44 \%$ des enseignants interviennent en milieu urbain, $36 \%$ le font en milieu rural et $20 \%$ en zone périurbaine. Les volumes horaires consacrés à l'activité d'enseignement varient de façon importante : $30 \%$ interviennent de 1 à $5 \mathrm{~h}, 28 \%$ entre 6 et $10 \mathrm{~h}, 19 \%$ de 11 à $15 \mathrm{~h}$ et $23 \%$ plus de $16 \mathrm{~h}$ par semaine.

Excepté pour $7 \%$ d'entre eux, les enseignants de judo partagent leur activité avec d'autres, au sein d'équipes de deux (13\%) à cinq enseignants (33\%), voire plus.

Les réponses indiquent que l'activité est tournée vers des publics très diversifiés (plusieurs réponses ont été cochées par les enquêtés), avec une dominante de jeunes de 5 à 12 ans ( $82 \%)$, puis d'adolescents (13-18 ans), jeunes adultes (19-30 ans) et adultes (31-50 ans) qui obtiennent chacun des scores de $78 \%$. Les cours de baby judo (60\%) occupent aussi une place importante, ainsi que ceux qui s'adressent aux licenciés de plus de 50 ans (25\%).

24 Enfin, on repère des conditions économiques contrastées entre Paris et la Province, repérables lorsque les enseignants changent de secteur ou de région : 
«Enseigner le judo en Province ou dans un club parisien, ce n'est pas la même chose. À Paris, tu es très bien payée, en province, j'ai été surprise, pas du tout. Tu dois bidouiller. " (Adèle, 38 ans). erreurs par rapport au geste le plus efficace, le plus juste» (3.47), «enseigner des techniques" (3.45), "préparer à la compétition » (2.99) reste moyennement mobilisée. Tout comme certains items relatifs aux savoirs nécessaires pour enseigner, à savoir le fait de bien connaître «la psychologie de l'enfant, de l'adolescent et de l'adulte» (3.37), ou « la biomécanique et la physiologie humaine" (3.15) qui semblent moins prioritaires, de même que le fait de « s'engager dans - ou de développer - des projets collectifs : fête du sport, téléthon, voyages» (2.97). Par ailleurs, il est intéressant de noter la manière dont les enquêtés positionnent l'enseignement du judo en termes de proximité avec d'autres activités ou métiers (3 réponses possibles). L'activité d'enseignant est massivement associée à celle d'un «éducateur » (83\%). Les rôles d' « entraîneur » (57\%) et de « sportif» (56\%) sont aussi convoqués. Enfin l'enseignant de judo se perçoit comme un «animateur » (45\%) ou un « technicien» $(40 \%)$. Des métiers tels que «guide» (31\%), "acteur social» (22\%) ou « comédien » $(7 \%)$ ne correspondent que de loin à la représentation que les enquêtés se font de leur activité d'enseignement.

De façon complémentaire, les entretiens réalisés avec les enseignants relatent tous des valeurs " historiques » du judo et de son caractère éducatif. Le code moral du judo ${ }^{6}$, la « culture judo» et ses valeurs sont considérés comme des outils éducatifs originaux qui permettent de fixer un cadre, des règles et une forme de respect de l'autre et de l'environnement. Ils permettent également de répondre aux attentes des parents qui inscrivent leur enfant au judo en espérant qu'au contact de l'activité sportive, considérée à partir de son arrière-plan symbolique et moral, il sera plus « cadré, éduqué ». 
«Le code moral, c'est quand même quelque chose grâce auquel on a autant de licences. Les enfants, ils nous les amènent pour, qu'entre guillemets, on les éduque » (Raphaël, 23 ans).

«Ce que je vais mettre beaucoup en avant c'est la culture judo, c'est-à-dire les valeurs. Je pense qu'on est dans une société où ça se perd. Je pense que l'utilité sociale du judo est très forte et que la fédération le sait » (Rémi, 41 ans). 2012) dans la culture judo. Mais plus encore, le judo apparaît pour certains enseignants comme une discipline de vie, une "école de vie », un « art de vivre » rendant compte de valeurs humaines fondamentales.

«Être judoka, c'est chercher un équilibre pour sa relation avec soi, pour sa relation avec les autres, avoir sa place dans la société et à partir de cette expérience, la plus riche et la plus ouverte possible, être capable d'adaptation » (Ange, 45 ans).

"Quand tu es judoka, tu es judoka à vie. Tu fais partie d'une tribu, d'une communauté particulière » (Adèle, 38 ans).

«Le judo est une discipline de vie ou une manière de vivre ou un art de vivre » (Pascale, 50 ans)

31 Ainsi, bien que les valeurs soient fortement présentes dans leur discours lorsqu'il s'agit de parler de leur pratique, les enseignants de judo doivent composer avec un ensemble d'éléments, parfois en tension, qui organisent leur activité au quotidien.

\subsubsection{Entre degré de satisfaction et difficultés liées à l'enseignement du judo}

$55 \%$ des enseignants interrogés se disent plutôt satisfaits de cette activité et $43 \%$ tout à fait satisfaits. Ces réponses peuvent être associées aux trois mots clés choisis par les enquêtés pour caractériser l'enseignement du judo aujourd'hui : «valeurs » (18\%), « éducation» (17\%), et " passion» (12\%) sont énoncées largement en tête. Viennent ensuite les termes de « pédagogie » $(9 \%)$, de « respect » $(9 \%)$ et de « partage » $(8 \%)^{7}$.

Malgré cette motivation à enseigner, des difficultés existent. Elles sont liées prioritairement à «l'évolution du judo » (2.7), aux « conditions de travail » (2.52), aux « tâches administratives à assurer » (2.49), au « contexte socio-économique ou culturel » (2.42). Les items choisis dans le questionnaire renvoient à un élargissement et une complexification de l'activité qui peut être vécue comme éloignée des motifs ayant présidé à l'engagement dans l'enseignement du judo.

On retrouve cette approche à partir des verbes fortement mobilisés par les enseignants de judo, dans le questionnaire (questions ouvertes) et dans les entretiens: «professerentraîner-éduquer-former-animer ». Les enseignant-e-s en soulignent les rapports ambigus, parfois même contradictoires. D'une part, ils défendent la richesse du judo et les multiples possibilités qu'il offre pour orienter/ajuster leur travail en fonction d'une demande sociale complexe qui vient parfois parasiter les logiques fédérales et sportives.

«Même pour mes compétiteurs, avant d'être un compétiteur, je sais qu'un jour ça va être des hommes et des femmes de demain...» (Rémi, 41 ans).

« Pour moi un enseignant c'est partager, transmettre des choses, mais il faut que les deux parties le fassent avec plaisir. Un enseignant ne doit pas donner d'ordres. » (Anaïs, 55 ans).

«Les enseignants de judo, on éduque, on forme... Le combat fait partie de la formation, c'est le but ultime » (Pascale, 50 ans). 
D'autre part, certains se démarquent, soulignant la spécificité de l'enseignement pour tous versus sélection et entraînement des meilleurs. Le verbatim suivant pointe des logiques différentes et sans aucun doute des tensions internes au groupe professionnel:

«Professer et entraîner ça n'a rien à voir... Professer, il faut éduquer et c'est important d'éduquer tout le temps pour moi. Les entraineurs sont plus carriéristes, qui vont chercher la réussite sportive de l'athlète pour justement se valoriser euxmêmes » (Rachel, 44 ans).

Derrière ces termes aux contours flous se jouent des manières de penser l'activité d'enseignement, de la vivre et de la faire vivre. L'enquête fait émerger un rapport à l'enseignement qui intègre à la fois une dimension praxéologique (valeurs), culturelle (rituels, techniques, grades), pédagogique (moyens mis en œuvre, gestion des groupes), mais aussi administrative (suivi des licences, des compétitions, etc.), avec en arrière-plan des logiques économiques plus ou moins affirmées.

\subsection{Les débats de valeurs et les logiques concurrentes : un système en tension inhérent à l'activité des enseignants de judo}

Les débats de valeurs et les logiques concurrentes rencontrées par les enseignants de judo sont énoncés dans leur discours sous la forme de tensions inhérentes à leur activité. Même si pour des raisons de présentation et d'écriture ces tensions sont présentées séparément, elles sont fortement imbriquées les unes aux autres.

\subsubsection{Entre " plaisir et effort », « loisir et compétition »}

Même si la notion de plaisir reste le vecteur essentiel de la pratique, le "goût de l'effort »" devient un véritable enjeu pour les enseignants de judo, plus particulièrement aujourd'hui, dans un contexte social où l'effort et l'activité physique sont, de leur point de vue, minimisés.

« L'enfant qui vient faire du loisir, il est là pour jouer. Son objectif c'est le plaisir. Le compétiteur, il y a tout le temps cet aspect de plaisir, mais avec un objectif de gagner » (Rémi, 41 ans).

«Se faire mal fait partie aussi de l'activité, aujourd'hui c'est important, car les enfants ne sont pas aussi actifs qu'avant » (Arthur, 40 ans).

Dans cette même idée de logiques concurrentes, «loisir et compétition" peuvent s'opposer, la compétition étant plutôt réservée dans les clubs aux enfants, le loisir aux adultes (sauf pour les clubs de haut niveau). Parfois ces deux notions se rencontrent, en particulier quand la compétition est elle aussi considérée par certains enseignants comme faisant partie de la pratique et comme un lieu singulier d'expériences.

«La compétition est réservée le plus dans les clubs aux enfants, les adultes viennent pour le loisir... » (Abdel, 36 ans).

«La compétition fait partie de la pratique... c'est une situation spécifique en contexte dans laquelle on doit développer son judo, l'adapter à l'adversaire » (Agathe, 44 ans).

« La compétition est quelque chose d'important, on doit s'y confronter dans le sens de gagner en expériences. Mais ce qui m'intéresse c'est le judo et la compétition comme conséquence du judo, comme faisant partie. Être judoka, ce n'est pas être compétiteur. On peut être les deux, mais pas au même moment » (Ange, 45 ans). tension concerne les exigences du haut niveau en judo et, par voie de conséquence, la 
perte potentielle de jeunes licenciés. En effet, avoir des judokas qui réussissent en compétition, c'est courir le risque de voir partir ces jeunes vers des instances fédérales (pôles espoirs, pôle France) et de perdre une dynamique construite au sein du club.

«Les enseignants de judo, on éduque, on forme... Le combat fait partie de la formation, c'est le but ultime. Pour autant, on ne va pas chercher la performance sportive à tout prix. On n'est pas dans la mentalité entraîneurs de haut niveau, qui casse avec cette idée de la viande qui sert de partenaire... Ce n'est pas vraiment ma philosophie. D'autant que beaucoup de mes élèves viennent avant tout pour se faire plaisir » (Abdel, 36 ans).

«Dans le haut niveau, les préoccupations sont autres. Tout le monde n'est pas là pour ton bien-être... » (Adèle, 38 ans).

« Le haut niveau, l'INSEP... c'est un autre monde. Nous on n'est pas sur les mêmes valeurs. Parfois avec le haut niveau en judo, tu te fais plus plaisir, les valeurs ne sont pas les mêmes. Là-haut c'est la performance à tout prix. On hésite à faire partir les jeunes » (Abdel, 36 ans).

41 Les ceintures et les grades en judo sont présentés par les enseignants comme un système qui permet de réconcilier différentes intentions éducatives en valorisant le travail de tous (judokas et enseignants).

« Le système des ceintures me plaît bien, ça me plaît bien parce que c'est un niveau de compétences techniques. Une ceinture, ça se mérite, donc on doit travailler, on l'obtient, on gravit des échelons. J'essaie de faire passer le message qu'on doit être comme ça dans la vie » (Rachel, 44 ans).

« Le passage de ceinture et de grade sont vraiment essentiels en judo. Ils attestent d'un niveau technique, d'un niveau d'engagement dans l'activité. On ne donne pas les ceintures, il faut travailler pour cela. L'enseignant et les élèves doivent travailler » (Abdel, 36 ans).

Pour échapper à ces tensions, chacun met en avant le travail comme étant une valeur sûre, comme un élément qui permet de dépasser les difficultés et contradictions rencontrées.

\subsubsection{Entre tradition et changement}

43 Le respect de la tradition, de la culture japonaise, de la méthode française pour enseigner le judo sont des éléments fortement revendiqués par les enseignants. Pour autant, les évolutions d'ordre pédagogique semblent aujourd'hui incontournables afin de s'adapter de façon optimale aux changements sociétaux.

«En judo classiquement, il y a la méthode française de judo, ça s'est préconisé par la fédération, mais il y a d'autres façons d'envisager l'apprentissage en judo » (Rachel, 44 ans).

«Il y a une évolution de la société et ne pas prendre en compte cette évolution, c'est une erreur. J'aime le judo traditionnel, le beau judo, le geste parfait... je suis fan du judo japonais, mais aujourd'hui les jeunes recherchent autre chose. On perd beaucoup d'ados en termes de licences donc il faut qu'on réponde plus à leur demande de spectaculaires, de jeux, de combats » (Adèle, 38 ans).

On perçoit aussi chez quelques un-e-s une volonté d'ouvrir le judo à des problématiques plus transversales, de sortir d'un cadre vécu comme trop étroit, dans une société en mouvement, qui nécessite des évolutions :

«Tu vois y'a pas que le judo dans la vie, aujourd'hui moi je profite, je fais plein de choses à côté... Même si je suis toujours une judoka, j'essaie de faire dans d'autres domaines tout ce que le judo m'a appris... pour le travail des appuis, la discipline, la 
gestion des blessures. L'idée, c'est d'enseigner des choses de façon plus transversale » (Anaïs, 55 ans).

\section{d'informations :}

«Par exemple, hier soir sur Facebook, j'ai donné des informations sur le stage avec mes élèves. Je trouve que c'est un bon outil de travail s'il est bien maîtrisé » (Rémi, 41 ans)

«Le club avait beaucoup évolué, j'avais un rôle de communication avec les adhérents, de les informer sur les compétitions, sur l'actualité du club... en me servant du numérique. C'est pour en général plus sur la partie organisation du club que sur la partie sportive » (Raynal, 35 ans)

" Mon projet est de créer le site du club... Aujourd'hui, la prise de licence se fait par internet dans tous les clubs. Donc rien que ça, ils ont le lien directement sur le site du club. En plus au lieu de passer par Facebook directement, ça ferait aller sur le site du club. Ça permettrait de mettre la pub, les sponsors sur le site... les jeunes sont tous les jours au téléphone, sur la page Facebook... » (Raphaël, 23 ans)

51 Le témoignage de Raphaël souligne la prise en compte des caractéristiques du public (élèves, parents, sponsors, etc.). Pour certains enseignants de judo, le numérique serait donc un élément devenu incontournable si l'on veut être réactif, en phase avec son époque et avec l'évolution des licenciés : 
« Maintenant avec tout ce qui est internet, c'est moi qui gère tout l'administratif. J'ai dû m'y mettre. Déjà la fédération s'est mise à l'extranet, donc il a fallu s'y mettre et j'ai quand même des jeunes dans le club et je sais que la communication passe par Facebook... On a fait un site internet. Les gens sont très contents parce que c'est très facile pour eux » (Salim, 38 ans).

Les entretiens permettent également d'identifier quelques dilemmes auxquels les enseignants de judo sont confrontés, tels qu'être partie prenante de l'environnement numérique sans être assurés de disposer localement des compétences requises :

\begin{abstract}
«Alors, il y a un site internet qui a été créé. C'est aussi comme ça que nous avons des personnes qui viennent nous voir, parce qu'elles nous ont vus sur le site internet. Malheureusement, et, on en discute depuis quelque temps, mais on ne sait pas comment faire, on aimerait utiliser Facebook. Parce qu'on s'aperçoit que Facebook est un vecteur intéressant, pour amener un public. On a discuté avec d'autres clubs qui l'utilisaient, et qui ont dit que c'est très bien. Donc, nous, on n'a pas de personnel qualifié pour s'occuper de ça. Et même la mise à jour de notre site nous pose toujours des problèmes, pour trouver une personne. Mais, on voudrait aller sur Facebook, effectivement. Enfin, entre autres, parce que, peut-être qu'il y a d'autres voies, que nous n'explorons pas, en ce moment et qui peuvent être intéressantes. On n'est pas fermé. On est bien ouvert, mais on n'a pas la compétence. On est là, mais on est un peu naïf » (Sirius, 66 ans).
\end{abstract}

Pour d'autres, des ressources locales existent et un partage des tâches peut s'organiser. Pour autant, il convient de rester vigilant sur de possibles dérives et de (se) donner des règles pour un usage optimal et maîtrisé :

«Alors, moi déjà, je n'ai pas Facebook. Déjà rien que pour moi, je n'aime pas trop les réseaux sociaux. Tout ce genre de choses, je n'y suis pas. Donc, la page Facebook, pour moi, c'est le président qui s'en occupe. Je fais passer des informations, je lui dis, tiens mets ça sur Facebook, et il s'en occupe » (Salvatore, 29 ans).

«Comment rendre compte de la vie et de l'évolution du club tout en ayant une approche de la communication qui préserve la vie privée de tous? Par exemple sur le Facebook de notre club, on ne met pas de photos c'est-à-dire les photos on les met sur notre site internet qui est protégé pour que les photos ne soient pas utilisées » (Rémi, 41 ans).

Enfin, quelques utilisations du numérique à visée pédagogique sont identifiées.

« Là, il y a eu les Jeux olympiques, quand il y a des tournois, on regarde les vidéos. Si on peut les suivre à la télé, c'est bien. Mais après, on regarde sur YouTube les vidéos. S'il y a des choses qui nous intéressent, on en parle entre nous. On va essayer de le travailler, de le décortiquer » (Salvatore, 29 ans).

55 Ainsi, on constate une prise en compte pragmatique des apports technologiques et, plus largement, l'inscription de l'enseignement du judo dans son époque. S'adapter aux évolutions est perçu comme indispensable. Pour autant, la question des valeurs, de la culture du judo reste prégnante et se trouve malmenée par des enjeux qui aujourd'hui s'invitent dans la sphère sportive et associative.

\title{
4.2.3. Entre rentabiliser une structure et préserver le sens de l'activité judo
}

Le judo est souvent mis en avant comme étant une activité sportive qui permet d'intégrer tous les types de publics. Il est considéré comme un " excellent support éducatif » pour tous les pratiquants, quels que soient leur âge ou leur sexe (Brousse, 2005 ; Groenen, 2005 ; Renaud, Monnin, \& Loudcher, 2011). La démocratisation et la diversification des pratiques apparaissent donc comme un levier essentiel pour le développement du judo. 
57 Selon les enseignants interrogés, cette diversification des pratiques dans les clubs a pour objectif prioritaire de faire entrer de nouveaux adhérents et de "faire des licences » (Juhle, 2012). Ainsi, diversifier apparaît comme une réelle nécessité économique pour rentabiliser la structure, mais également pour la dynamiser et la développer. Ces enjeux économiques se situent parfois en tension avec «l'esprit du judo». Une forme de vigilance est mise en avant par les enseignants, afin de préserver le sens premier de l'activité judo.

«À la base, il y avait des cours de judo trois fois par semaine et j'ai rajouté des créneaux de Taïso, c'est des aptitudes de judo, des déplacements de judo, du renforcement musculaire, de la coordination, de l'équilibre, tout ce qui s'adresse au judo, mais sans opposition. J'ai beaucoup de personnes âgées qui ont peur de la chute et donc ça, c'est super » (Raphaël, 23 ans).

«Le baby judo, c'est plus basique, c'est moins de l'activité judo, c'est vraiment de l'éveil corporel » (Raynal, 35 ans).

«Aujourd'hui pour continuer à vivre, on a besoin de diversifier nos pratiques. Le judo OK, mais il y a d'autres disciplines, le Taïso par exemple, et d'autres disciplines de combat... même si la fédération ne voit pas ça d'un bon œil. On est obligé de proposer d'autres disciplines pour maintenir des adhérents » (Barnabé, 58 ans).

Cette approche est plus prégnante chez les jeunes qui doivent avoir un volume d'heures suffisant pour assurer un travail rémunéré à plein temps. Le fait de diversifier les pratiques dans le club et/ou de travailler avec plusieurs clubs permet à certains enseignants de judo de créer et pérenniser leur emploi. Ainsi, être éducateur sportif semble pour certains, être une voie de reconversion professionnelle (Julhe \& HascharNoé, 2010) permettant d'allier passion et métier.

«C'était un club bénévole à la base, donc on ne pensait pas un jour payer un professionnel là-bas... mais de fil en aiguille il y avait un projet à faire sur le BPJEPS et mon projet, c'était de devenir payé sur ce club. L'idée de devenir pro à plein temps est devenue réelle " (Arthur, 40 ans).

" Je suis salarié du club, mais j'ai plusieurs casquettes et plusieurs employeurs. Et ça me fait un plein temps. Je fais judo du lundi au dimanche » (Abdel, 36 ans).

«Aujourd'hui, j'essaie de faire en sorte d'avoir un plein temps en judo. J'ai commencé en étant bénévole, mais bon là j'ai besoin de vivre. Le bénévolat, c'est bien un temps, mais là j'ai des compétences et je veux essayer d'allier ma passion avec mon travail » (Armand, 27 ans).

Cependant, passer du bénévolat à une activité salariée n'est pas une chose aisée et demande un engagement professionnel qui met en jeu d'autres compétences techniques, notamment des compétences de chef de projet et de gestionnaire.

« Être enseignant de judo, c'est avoir plusieurs casquettes enseignant, animateur, éducateur, coach, entraîneur, chef de projet » (Adèle, 38 ans).

" Aujourd'hui, en plus d'être enseignant de judo, je suis quasiment chef d'une petite entreprise. Il faut que j'enseigne, que je cherche de l'argent, que je travaille avec plein de partenaires institutionnels... Il faut vraiment d'autres compétences que celle simplement à enseigner » (Ange, 45 ans).

60 Ces enjeux économiques génèrent le plus souvent un surcroit de travail qui modifie le périmètre de l'activité d'enseignement, combinée à d'autres registres d'intervention (communication interne et externe, recherche de subventions, création d'évènements, etc.). Dynamiser le club dans l'espace social, le faire exister au plan des résultats sportifs, accueillir des publics diversifiés aux attentes spécifiques renforce la charge de travail visible et invisible. Le cœur de l'activité enseignante se déporte et se déploie. Pour les enseignants, cette situation vient parfois bousculer les équilibres de vie. 


\subsubsection{Trouver un équilibre entre vie professionnelle et vie privée}

61 Selon le modèle de Curie \& Hajjar (1987), les échanges entre la vie de travail (sphère professionnelle) et la vie hors travail (sphères familiale, sociale et personnelle réunies) sont plutôt considérés par les enseignants de judo comme étant une aide. Ainsi $62,7 \%$ des enseignants de judo affirment que la sphère personnelle est une aide pour leur vie professionnelle et parallèlement $66,4 \%$ disent que leur sphère sociale est aussi une aide à leur vie professionnelle. En revanche, les échanges « vie professionnelle et vie familiale » semblent plus complexes et considérés par $23,6 \%$ des enseignants de judo comme étant un obstacle.

Cette relation vie de travail/vie hors travail renvoie autant à des ressources qu'à des contraintes pour les enseignants de judo, mais dans la sphère hors travail, la sphère familiale est la plus difficile à concilier avec les trois autres. Parmi les ressources identifiées nous trouvons, entre autres, la passion du métier, l'enrichissement et l'épanouissement personnel liés à l'activité judo dans toutes ces dimensions, mais aussi le partage et les échanges avec toutes les personnes du club. Les contraintes concernent plus particulièrement les déplacements, les horaires, l'éloignement de la famille.

On constate que l'équilibre professionnel et familial est difficile à trouver, et donne lieu à des modes de régulation singuliers. Une conciliation est possible entre les deux sphères grâce à la « famille judo » ou à un « arrangement » au sein du couple.

« Toute la famille est dans le judo, donc c'est beaucoup plus facile. Mon mari fait la même chose que moi. Les enfants comprennent parce qu'elles connaissent très bien l'activité. Sur le séminaire on n'est pas avec nos enfants. Mais en même temps je suis là, je me régale. Quand je partage, j'adore ça. Mais c'est difficile pour une femme » (Rachel, 44 ans).

« Ma femme est aussi prof de judo, mais à la naissance des enfants elle a décroché. On s'accorde des moments. Des fois elle vient avec moi en compétition avec les enfants. J'ai fixé le lundi soir, le lundi soir de ne pas faire cours pour être justement avec mes enfants, pouvoir faire les devoirs avec mes enfants et rester avec ma femme. Et mes horaires de travail permettent, aussi parce que j'ai des trous dans la journée, d'aller les chercher à la cantine et de manger avec eux le midi» (Abdel, 36 ans).

64 À l'inverse, la régulation avortée et un conflit insurmontable peuvent donner lieu à des décisions émotionnellement compliquées et parfois irréversibles :

«Oui le temps qu'on a passé, entre midi et deux, à courir, lorsque je ne rentrais pas à la maison, ma fille ne me voyait pas et je ne la voyais pas. Quand je rentrais le soir, je ne la voyais pas non plus, c'était $10 \mathrm{~h} 00$. Le matin, je ne la voyais pas parce qu'elle n'était pas levée. Donc je la vois maintenant alors qu'elle a 35 ans... » (Brice, 67 ans).

«C'est ingérable, le couple explose... » (Baptiste, 67 ans).

Les jeunes enseignants semblent lucides et conscients de cette difficulté :

«Parce qu'on n'a pas encore d'enfant, je ne le sais pas, je vous le dirai quand on en aura, c'est en projet. Parce que je pense que c'est compliqué. Il faut choisir à un moment parce qu'en plus avec le boulot que je fais, si le week-end je ne peux pas me poser avec ma famille tout ça, ou que je suis en compétition, en stages, cela demandera de se répartir les tâches, d'avoir quelque chose de plus carré, organisé. Pour ne pas sacrifier l'un ou l'autre, il y en a qui ont laissé leur couple dans tout ça » (Bastien, 33 ans). 
L'enquête montre que le rythme de travail à " contre-courant » du rythme «classique » est également générateur de conflits et de régulations temporelles, ce qui engendre soit une tentative de cloisonnement des activités: "Je ne mélange pas du tout le travail avec le judo. D'ailleurs, j'ai le travail et le judo. Les deux fonctionnent ensemble, mais côte à côte» (Béatrice, 30 ans), soit une gestion réfléchie et planifiée du temps en fonction de l'activité principale :

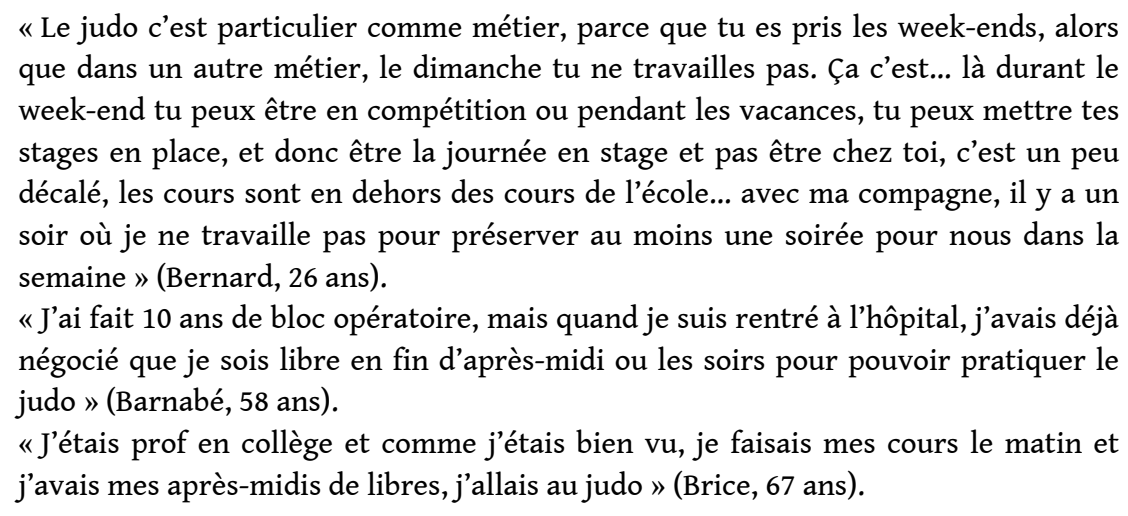
que dans un autre métier, le dimanche tu ne travailles pas. Ça c'est... là durant le week-end tu peux être en compétition ou pendant les vacances, tu peux mettre tes stages en place, et donc être la journée en stage et pas être chez toi, c'est un peu décalé, les cours sont en dehors des cours de l'école... avec ma compagne, il y a un soir où je ne travaille pas pour préserver au moins une soirée pour nous dans la semaine » (Bernard, 26 ans).

«J'ai fait 10 ans de bloc opératoire, mais quand je suis rentré à l'hôpital, j'avais déjà négocié que je sois libre en fin d'après-midi ou les soirs pour pouvoir pratiquer le judo » (Barnabé, 58 ans).

«J'étais prof en collège et comme j'étais bien vu, je faisais mes cours le matin et j'avais mes après-midis de libres, j'allais au judo » (Brice, 67 ans).

Ainsi, l'agencement vie de travail-vie hors travail reste difficile à gérer, chaque sphère risquant d'interférer sur l'autre. En conséquence, si les relations sociales sont riches, elles tendent à se déployer uniquement dans le monde du judo :

«On aime bien se retrouver entre profs de judo les week-ends où on n'a pas de compétitions. On se comprend, c'est plus simple et là on partage des choses sur les formations que les uns ou les autres ont eues, ou encore les informations que la fédé a fait passer » (Sacha, 40 ans).

L'enquête met donc en avant un équilibre difficile entre les différentes sphères de la vie familiale, personnelle, sociale et professionnelle. La forte implication dans le judo crée des tensions que chacun tente de surmonter, en mobilisant des ressources ou des stratégies relativement diversifiées.

Nous allons à présent revenir sur l'ensemble des résultats et tenter de comprendre comment les enseignants de judo parviennent à dépasser le système de tensions repéré dans l'étude pour construire un monde relativement cohérent dans lequel la " passion du judo ", largement revendiquée, conserve une place centrale.

\section{Discussion}

\subsection{Une activité organisée autour de valeurs fondatrices}

L'enseignement du judo est soumis à un certain nombre de tensions, interdépendantes, dont témoignent les enseignants interrogés. Les deux premières tensions (plaisir vs effort et tradition $v s$ changement) renvoient plus particulièrement à la dimension pédagogique ; l'enseignant est amené à faire des choix vis-à-vis des publics, mais aussi des enjeux sportifs et éducatifs de cette activité sportive. Les deux tensions suivantes (rentabilité vs sens de l'activité et équilibre professionnel vs équilibre de vie) rendent davantage compte de dimensions économiques et sociales. Ce système de tensions, sans cesse à orchestrer pour conserver le sens de l'action, est fortement relié aux contextes d'intervention et aux formes d'emploi (statut salarié/bénévole, travail dans un seul ou plusieurs clubs, etc.). 
71 Au-delà de ces tensions, l'enquête met en avant un rapport au travail positif chez la grande majorité des enseignants de judo et un fort attachement aux valeurs partagées et culturellement définies par les fondateurs de cette activité (Brousse, 2005). Ces valeurs éducatives du judo nourrissent les discours et semblent permettre, in fine, le dépassement des tensions. Il s'agit avant tout de former un citoyen et de positionner l'enseignement du judo comme une « école de vie », à travers un "Code moral » auquel chacun reste fidèle, sur et hors du tatami, dans le sport comme dans la cité. Le monde du judo, porté par des principes d'altérité, fonde le sens de l'activité, donnant aux enseignants un sentiment de fierté. Dans les propos recueillis (questionnaire et entretiens), les valeurs du judo donnent le cap dans un environnement social jugé en perte de repères; elles constituent une balise pour les jeunes licenciés que les plus anciens ont en charge de maintenir, parfois contre vents et marées.

72 Par ailleurs, ces valeurs partagées et défendues, structurantes, participent à la construction d'une image sociale. Selon de Gauléjac et Taboada-Léonetti (1994), pour exister socialement, chacun doit faire la preuve de ses compétences, de son utilité, de ses qualités et être reconnu pour son travail, qu'il soit rémunéré ou pas. Ainsi, la «valeur travail» prend une importance capitale, car elle participe d'un processus d'insertion sociale : l'intégration par le travail (rémunéré ou non) est économique, symbolique et sociale :

« économique, car elle autorise l'insertion et la participation au travers d'activités de production et de consommation; sociale parce qu'elle entraîne la constitution de liens sociaux par l'inscription dans des groupes; symbolique par les normes et valeurs communes qui sont socialement construites » (Royer, 2002, p. 24),

Enseigner le judo, c'est donc trouver sa place dans la société et être reconnu comme œuvrant à la construction du futur citoyen. C'est faire en sorte que cette activité soit valorisée pour ce qu'elle est et pour ce qu'elle permet. Orienté par cette finalité, l'enseignement devient un espace d'investissement puissant qui nécessite un engagement dans la durée.

\subsection{Une implication professionnelle en lien avec les finalités éducatives du judo}

Malgré les tensions que peuvent vivre les enseignants, le judo est d'abord envisagé comme une philosophie de vie. La passion pour une activité sportive, pratiquée depuis plusieurs années, traverse les processus de transmission qui s'orientent vers une éducation globale et complète de l'individu, y compris dans sa visée compétitive. Les relations construites au sein du club avec et dans la communauté de pratiquants, en lien avec des valeurs partagées, contribuent à des formes d'accomplissement (Deci \& Ryan, 1985) dont témoignent les enseignants de judo.

Dans leur activité « déclarée », ces derniers mettent en avant le plaisir de découvrir les élèves (enfants, adolescents ou adultes), de les accompagner et de participer à leur transformation physique, mentale et morale. Ce rapport affectif entretenu avec les fonctions qu'ils assument (Paugam, 2000) génère des formes d'implication organisées autour du triptyque « sens-repères-contrôle » proposé par Mias (1998). De fait, le judo fait sens dans la mesure où les enseignants retrouvent, exploitent, transmettent des techniques, des attitudes et des valeurs intégrées depuis longtemps dans leur propre pratique. Par ailleurs, la fidélisation au club qui les a formés permet la construction de 
nombreux repères dans l'environnement professionnel et personnel: repères pour l'action, repères psycho-sociaux plus larges qui les positionnent au cœur des problématiques éducatives. Les enseignants interrogés revendiquent une réelle place dans l'espace du judo et plus largement dans l'espace social (liens avec les familles, l'école, les municipalités, etc.). En ce sens, ils gardent un sentiment de contrôle de la situation, entendu ici dans le sens de possibles marges de manœuvre pour l'action. Si des normes existent et cadrent l'activité d'enseignement, elles semblent relativement bien acceptées, du moins dans les discours.

\subsection{Entre activité, expérience et contexte : un rapport au travail réajusté}

Le rapport au travail des enseignants de judo (Charlot, 1997) inclut des représentations, des valeurs qui organisent la pratique et lui donnent un sens. Il est entendu comme le " rapport affectif que les individus entretiennent avec les fonctions qu'ils exercent dans la sphère du travail » (Paugam, 2000, p. 43). Comme nous l'avions évoqué supra, le degré de satisfaction est en lien avec les niveaux d'engagement revendiqués/consentis, qui alimentent un processus de reconnaissance sociale.

77 Les enseignants de judo revendiquent - et parfois déplorent - une activité composite, qui se déploie à la fois face aux élèves, mais aussi avec différents interlocuteurs (parents, structures fédérales, municipales, etc.). En ce sens, ils doivent orchestrer un certain nombre de fonctions (de rôles), comme les enseignants de l'éducation nationale, à ceci près que les élèves qui viennent pratiquer dans la durée ont choisi le judo et lui attribuent une valeur particulière. Par ailleurs, les enseignants ont à s'adapter de façon singulière aux évolutions du judo, des publics, du contexte local, tout en prenant en compte les directives nationales.

78 Ainsi, l'activité d'enseignement est complexe à gérer en raison de son côté chronophage, qui vient perturber les équilibres familiaux et sociaux. Ceci peut créer des dilemmes que chacun tente de dépasser en activant tel ou tel registre (technique, pédagogique, relationnel, moral, politique, etc.), car enseigner le judo, c'est agir avant tout. Un déplacement/réajustement s'opère des valeurs déclarées aux valeurs « en acte » (Perez et Perez-Roux, soumis) pour faire face aux - et pour faire avec les - tensions inhérentes à l'activité.

La manière d'appréhender le travail suppose donc des choix raisonnés et s'inscrit dans l'ordre d'une rationalité contingente qui vise non pas « la » solution optimale, mais plutôt celle qui est satisfaisante et qui convient à l'action contextuelle (Simon, 1997).

\section{Conclusion}

80 L'étude souligne les tensions que peuvent vivre les enseignants de judo dans leur activité, que celle-ci soit rémunérée ou pas. En effet, ces derniers sont au carrefour de plusieurs logiques, parfois délicates à tenir conjointement, car elles génèrent des dilemmes et supposent des choix tant professionnels que personnels. Ceci rappelle de façon troublante ce que disent Tardif et Lessard (1999) à propos des enseignants du monde scolaire :

« l'enseignant est une sorte de caméléon professionnel qui doit sans cesse changer de peau et jouer plusieurs personnages: celui du pédagogue préoccupé du climat positif de sa classe et des relations humaines instaurées et maintenues tout au long 
de l'année avec chacun des élèves sous sa responsabilité; celui du didacticien soucieux de transmettre sa matière, de couvrir le programme et de favoriser l'apprentissage des contenus ; celui du maître tranchant les conflits entre élèves ou entre les élèves et lui; celui du père ou de la mère de famille arbitrant les problèmes de discipline ; celui du gardien ou du policier ramenant l'ordre ; parfois celui de l'ami ou du confident établissant une relation d'aide ou de soutien avec certains élèves en butte à des problèmes personnels, affectifs, existentiels, familiaux, etc. ». (p. 131).

81 Bien que les contextes de pratique et que les motivations des publics soient sensiblement différents (obligation scolaire/choix d'une activité en loisir), on constate que les problématiques de l'enseignement, quels que soient les terrains (scolaire, sportif, santé, travail social, etc.) peuvent se rejoindre sur certains aspects. Est-ce en partie pour cela que les éducateurs sportifs de judo (statut officiel) se définissent comme enseignants et se revendiquent, bien au-delà de leur discipline sportive, comme des éducateurs soucieux de former un citoyen lucide, responsable et respectueux d'autrui ? Les discours s'organisent autour de l'utilité et de la reconnaissance sociale qui donne aux enseignants de judo leur légitimité. Pour autant, cette façon d'être, de vivre et de s'inscrire dans la cité en transmettant des valeurs semble aujourd'hui bousculée par l'évolution des contextes sociaux, économiques, institutionnels et humains. Cela nécessite de construire un monde cohérent, consistant et "protégé » par des normes partagées, mais ouvert sur des compromis provisoirement acceptables.

\section{BIBLIOGRAPHIE}

Balleux, A., \& Perez-Roux, T. (2011) (Eds.). Transitions professionnelles et recompositions identitaires. Recherches en éducation, 11.

Barrère, A. (2002). Les enseignants au travail. Routines incertaines. Paris : L'Harmattan.

Bergeret, R. (1983). La relation maître-disciple. Sa présence dans le judo, son rapport à la voie dans les arts martiaux. Thèse de doctorat non publiée, Université des Sciences sociales, Grenoble, France.

Blanchet, A., \& Gotman, A. (2007). L'enquête et ses méthodes : l'entretien. Paris : Nathan.

Bouthier, D. (2000) Contribution des recherches didactiques en sports de combat. In A. Terrisse (Ed.), Recherches en Sports de Combat et en Arts Martiaux, État des lieux, (p. 95-109). Paris : Éditions Revue EP\&S.

Brousse, M. (2005). Les racines du judo français : histoire d'une culture sportive. Bordeaux : PUB.

Bui-Xuan, G. (2011). Les sports de combat comptent parmi les plus éducatifs (Préface). In J.-F. Loudcher \& J.-N. Renaud (Eds.), Éducation, sports de combat et arts martiaux (p. 7-14). Grenoble : PUG.

Charlot, B. (1997). Du rapport au savoir. Paris : Anthropos.

Cleret, B. (2013). L'ethnographie comme démarche compréhensive : immersion dans les dynamiques consommatoires du rap en France. Recherches Qualitatives, 32(2), 50-77. 
Collinet, C., Bernardeau., \& Moreau, D. (2009). Les éducateurs sportifs en France depuis 1945. Rennes : PUR.

Curie, J., \& Hajjar, V. (1987). Vie de travail - Vie hors travail : la vie en temps partagé. In C. LévyLeboyer, \& J.C. Sperandio (Eds.), Traité de Psychologie du travail (p. 37-55). Paris : PUF.

Deci, E. L., \& Ryan, R. M. (1985). Intrinsic motivation and self-determination in human behavior. New York : Plenum.

Dubar C. (2000). La crise des identités. L'interprétation d'une mutation. Paris : PUF.

Dubar, C., \& Tripier, P. (1998). Sociologie des professions. Paris : Armand Colin.

Dutercq, Y. (2008). Former des élites dans un monde incertain : stratégies scolaires et recherche de justice. Éducation et sociétés, 21, 5-16.

Dutercq, Y., \& Perez-Roux, T. (2011). La démocratisation de l'accès aux grandes écoles : vers une nouvelle professionnalité enseignante ? Recherche et formation, 67, 135-148.

Fleurance, P., \& Perez, S (2006). Les référentiels professionnels et de formation des entraîneurs nationaux "Chef de projet performance ». Rapport de recherche non publié, Paris : Ministère de la Jeunesse et des Sports, Direction des Sports \& Délégation à l'Emploi et aux Formations

Fleurance, P., \& Perez, S. (2008). Étude sur « le DTN» - «la DTN». Rapport de recherche, Direction des Sports, Paris : INSEP. Récupéré du site $:$ http://pfleurance.hautetfort.com/list/rapports-auministere-des-sports/3-analyse-de-l-activite-du-directeur-technique-national-et-d.html

Fleurance, P., \& Perez, S. (2009). Analyse des métiers de CTS ayant moins de 50 \% de missions d'entraînement. Rapport de recherche, Ministère de la Jeunesse et des Sports, Direction des Sports, Paris : INSEP. Récupéré du site : http://pfleurance.hautetfort.com/list/rapports-au-ministeredes-sports/4-pre-rapport-sur-l-analyse-du-metier-de-cts-ayant-moins-de.html.

Forquin, J-C. (1994). Valeurs. In P. Champy \& C. Eteve (Eds.), Dictionnaire encyclopédique de l'éducation et de la formation (pp. 1025-1029). Paris : Nathan.

Gauléjac, V (de), \& Taboada-Léonetti, I. (1994). La lutte des places. Paris : Epi.

Gaudin, B (2009). La codification des pratiques martiales : une approche socio-historique. Actes de la recherche en sciences sociales, 179(4), 4-31.

Groenen, H. (2005). L'ukemi, le randori et le kata. Une histoire culturelle des méthodes d'entraînement en judo : étude comparée France Belgique de l'entre-deux-guerres à la fin des années 1950. Thèse de doctorat non publiée, Université de Lyon 1, Lyon, France.

Julhe, S. (2012). Le judo et l'enfant. Regards et pratiques d'enseignants. STAPS, 96-97, 121-137.

Julhe, S., \& Haschar-Noé, N. (2010). Trajectoires et stratégies professionnelles dans le secteur de l'animation sportive : le cas des enseignants d'arts martiaux. Sociétés Contemporaines, 77(1), 7-29.

Kaufmann, J-C. (1996). L'entretien compréhensif. Paris : Armand Colin

Laplantine, F. (1996). La description ethnographique. Paris : Nathan université.

Loizon, D., \& Martin, D. (2006). Des savoirs enseignés à l'expérience des enseignants : un exemple de mutualisation chercheur-praticien. STAPS, 72(2), 107-118.

Mazade, O., \& Hinault A-C. (2014) (Eds.). Les identités professionnelles à l'épreuve des transitions. Sociologies pratiques, $28,3-8$.

Mias, C. (1998). L'implication professionnelle dans le travail social. Paris : L'Harmattan.

Moscovici, S. (1961). La psychanalyse, son image et son public. Paris : PUF. 
Paugam, S. (2000). Le salarié de la précarité. Paris : PUF.

Perez, S. (2009). Cognition et formation en sport de performance : De nouveaux cadres de pensée pour comprendre l'activité et la formation des cadres du sport de haut niveau ? Intellectica, 2(52), 119-137.

Perez, S., \& Perez-Roux, T (soumis). Comprendre l'activité des enseignants de judo : des valeurs déclarées aux valeurs « en acte ». Recherches en éducation.

Perez-Roux, T. (2011). Identité(s) professionnelle(s) des enseignants : les professeurs d'EPS entre appartenance et singularité. Paris : Editions EP\&S.

Perez-Roux., T., \& Balleux, A. (2014) (Eds.). Mutations dans l'enseignement et la formation : brouillages identitaires et stratégies d'acteurs. Paris : L'Harmattan.

Perez-Roux, T., \& Salane. F. (2013) (Eds.). Identités professionnelles en crise(s) ? Recherche et formation, 74 .

Royer, D. (2002). Qu'en est-il de la valeur travail dans notre société contemporaine ? Empan, 46(2), 18-25.

Renaud, J.-N., Monnin, E., \& Loudcher, J.-F. (2011). Le judo comme éducation physique ? Kano et Coubertin face à la nouvelle marche du monde. In J.-F. Loudcher \& J.-N. Renaud (Eds.), Éducation, sports de combat et arts martiaux (p. 133-149). Grenoble : PUG.

Simon, H.A. (1997). Models of bounded rationality. Tome III, Cambridge. MA : The MIT Press. SOFRES (2001). Professeurs de judo. Rapport de Synthèse pour la Fédération Française de Judo. Paris.

Tardif, M., \& Lessard, C. (1999). Le travail enseignant au quotidien. Expérience, interactions humaines et dilemmes professionnels. Bruxelles : De Boeck

Terrisse, A. (1996). Analyse de la transposition didactique en judo : évolution du "savoir combattre" dans l'enseignement du judo à l'école à travers la revue EPS de 1950 à 1993. Revue française de pédagogie, 116(1), 65-75.

Van Zanten, A. (2010). L'ouverture sociale des grandes écoles : diversification des élites ou renouveau des politiques publiques d'éducation? Sociétés contemporaines, 79(3), 69-95.

\section{NOTES}

1. Fédération Française de Judo, jujitsu, kendo et Disciplines Associées.

2. Dans cet article, nous avons opté pour une écriture générique qui englobe tant les hommes que les femmes.

3. Dans le cadre de l'enseignement du judo, le travail peut être rémunéré ou pas. Nous entendons par travail non rémunéré, les activités non rétribuées qui pourraient théoriquement être accomplies contre rémunération par une tierce personne: travail domestique et familial, activités honorifiques et associatives (travail bénévole organisé), etc.

4. Chaque année, la Fédération Française de Judo organise le Colloque National des Haut-Gradés qui regroupe les judokas, de niveau ceinture noire $6^{\mathrm{e}}, 7^{\mathrm{e}}, 8^{\mathrm{e}}$ et $9^{\mathrm{e}}$ Dan.

5. Selon l'étude SOFRES (2001), un enseignant de judo avait en moyenne 19 ans d'ancienneté dans son activité et deux enseignants sur trois souhaitaient la continuer. L'enseignement du judo était diversifié autour de trois pratiques distinctes (initiation, entraînement, perfectionnement) et s'adressait à tous les types de publics (enfants, adolescents et adultes). Les valeurs d'éducation (31\%) et d'enseignement (19\%), le respect (18\%) étaient mises en avant, par les enseignants, à 
travers le rôle éducatif du judo. Enfin, ces derniers enseignaient essentiellement le judo dans un seul club, avec un seul employeur pour la majorité d'entre eux.

6. Le Code moral du judo s'organise autour de six qualités fondamentales: amitié, courage, modestie, respect, contrôle de soi, politesse (https://www.ffjudo.com/les-valeurs).

7. Les autres termes semblent davantage polysémiques : «patience » $(7 \%)$, «transmission » (6\%) et «discipline » $(5 \%)$ sont aussi présents, avant une liste de nombreux mots, qui n'ont pas encore été agrégés au niveau sémantique.

8. Le développement du "goût de l'effort » s'inscrit dans les finalités de l'Education Physique et Sportive à l'école, au collège et au lycée ; il constitue un enjeu pour/dans les pratiques sportives. Les sites internet des clubs de judo en font une valeur importante. Ce terme, de sens commun, est donc très usité dans le milieu que nous avons étudié.

9. Les arts martiaux mixtes ou mixed martial arts (MMA), sont un sport de combat complet, associant pugilat et lutte au corps à corps. Les deux combattants peuvent utiliser de nombreuses techniques; selon les fédérations, sont autorisées les techniques de percussion telles que coups de pied, de poing, de genou et de coude, mais aussi les techniques de corps à corps debout (clinch), de projections et de soumission (grappling) et quelquefois des techniques particulières de percussion au sol. Ses détracteurs soulignent la violence des coups qui peuvent être portés avec peu de contrôle lorsqu'un combattant se situe au sol. Ses partisans admettent cette violence grâce à la diversité des zones de frappes possibles et la possibilité d'aller au sol (Dans Wikipédia, repéré à https://fr.wikipedia.org/wiki/Arts_martiaux_mixtes).

\section{RÉSUMÉS}

Les évolutions socio-économiques, idéologiques, politiques qui affectent aujourd'hui les champs de l'enseignement, de la formation et de l'éducation, conduisent les acteurs concernés à requestionner le sens de leur travail. La présente contribution s'intéresse aux représentations et valeurs des enseignants de judo. Il s'agit de repérer comment ces derniers se positionnent vis-àvis des logiques parfois contradictoires auxquelles ils doivent répondre. L'étude s'inscrit dans une double orientation : psycho-sociale (représentations, valeurs, implication professionnelle) et sociologique (rapport au travail, reconnaissance). Au plan méthodologique, suite à une approche ethnographique, deux séries d'entretiens semi-directifs ont été conduites en 2016 par une équipe de quatre chercheurs sur la région Occitanie. Par ailleurs, un questionnaire (sous sphinx) a été proposé en octobre-novembre 2016, sur la même région, puis diffusé au niveau national. Le croisement de ces différentes données permet de comprendre les valeurs partagées et les tensions auxquelles un certain nombre d'enseignants de judo sont confrontés dans leur activité au quotidien. Les résultats soulignent et illustrent les nombreux dilemmes à gérer : entre plaisir et effort, loisir et compétition, tradition et changement. Les enseignants de judo doivent veiller de plus en plus à rentabiliser leur structure, sans perdre pour autant les valeurs fondatrices du judo. Ils ont aussi à trouver un équilibre entre vie professionnelle et vie privée. L'étude éclaire, au moins pour partie, le rapport au travail que ces enseignants construisent pour s'adapter aux évolutions et conserver le sens de leur action.

Today's socio-economic, ideological and political changes affecting the fields of education and training lead the relevant actors to question the meaning of their work. This contribution focuses on the representations and values of judo instructors. Its aim is to identify how they deal with 
the occasionally contradictory forms of logic underpinning their activity. The background of the study is twofold: psycho-social, relating to representations, values and professional involvement, and sociological, relating to perception of work and professional recognition. At the methodological level, following an ethnographic approach, two series of semi-directive interviews were conducted in 2016 by a team of four researchers in the Occitanie area. In addition, data was collected in October-November 2016, during a professional meeting and later online from a self-administered questionnaire. The analysis of the compiled data allows us to gain a better understanding of the shared values and the tensions many judo instructors are faced with in their day-to-day activities. In particular, the results highlight and illustrate the range of dilemmas instructors have to deal with, between pleasure and effort, leisure activity and competition, tradition and change. Judo instructors increasingly have to ensure the profitability of their activity structure, but at the same time without losing the fundamental values of judo and while balancing family and career. The study sheds light, at least in part, on the perception of work that these instructors build in order to adapt to changes and to maintain the meaning of their activity.

\section{INDEX}

Mots-clés : mutations, rapport au travail, représentations, valeurs, activité, enseignants de judo Keywords : social changes, perception of work, perceptions, values, activity, judo educators

\section{AUTEURS}

\section{THÉRÈSE PEREZ-ROUX}

Université Paul-Valéry Montpellier 3, Laboratoire Interdisciplinaire de Recherches en Didactique Education et Formation (LIRDEF EA3749) - therese.perez-roux@univ-montp3.fr

\section{SYLVIE PEREZ}

Université de Montpellier, LIRDEF EA3749 - sylvie.perez@umontpellier.fr

\section{PHILIPPE GABRIEL}

Avignon Université, LIRDEF EA3749 - philippe.gabriel@univ-avignon.fr

\section{JACQUELINE PAPET}

Université de Montpellier, LIRDEF EA3749 - jacqueline.papet@umontpellier.fr

\section{FLORENCE CASSIGNOL}

Université Perpignan Via Domitia (UPVD), Laboratoire Européen Performance Santé Altitude (LEPSA, EA4604) - florence.cassignol@univ-perp.fr 